\title{
UNA DESCONSTRUCCIÓN HISTÓRICA Y SOCIAL DE LA NOCIÓN DE CENTRO HISTÓRICO*
}

\author{
Miguel Martínez López
}

\section{RESUMEN}

En este trabajo se revisan distintas concepciones sobre los centros históricos y su doble utilidad: tanto para hacer de ellos un objeto de investigación de una o más disciplinas académicas; como para fundamentar las intervenciones urbanísticas y sociales que sobre ellos se vienen realizando en las últimas décadas. El análisis parte de un punto de vista sociológico para preguntarse en qué medida las concepciones sobre los centros históricos de las ciudades de tamaño medio y de metrópolis están eludiendo los problemas sociales que en ellos se alojan.

En líneas generales se puede distinguir una evolución en los discursos dominantes sobre el centro histórico que abarca desde posturas demoledoras e higienistas de renovación urbana, hasta una concepción conservacionista, primero, estigmatizadora socialmente después y, finalmente, «estratégica» o mercantil, en coherencia con la reestructuración económica que se le impone últimamente a las ciudades.

En el caso que he podido investigar a fondo —el Casco Vello de Vigo (Martínez, 1995, 1996) - y en la comparación con otros cascos antiguos, he podido verificar estos desplazamientos. Pero también, el proceso de desconstrucción y duda metódica sobre los sentidos y connotaciones que adquieren los centros históricos, puede revelar que entre unas experiencias de unas ciudades a otras no existe una nítida transacción o copia de modelos —especialmente de aquellos más progresistas - y que debajo de las distintas nociones dominantes queda toda una heterogeneidad oculta que no se suele considerar dentro de las políticas rehabilitadoras: problemas sociales de vivienda y agrupamientos, segregaciones espaciales, la historia de la participación ciudadana, etc.

Palabras clave: Sociología, Urbanismo, Rehabilitación, Centros Históricos.

\begin{abstract}
In this article I suggest that many social problems are hidden with the common concepts of 'historical centres' (the inner city). In order to analize the different meanings

\footnotetext{
* Este artículo es una revisión de un fragmento de la Tesina de Licenciatura presentada en la Universidad Complutense de Madrid, en el Dpto. de Ecología Humana y Población, septiembre de 1996: «El dominio histórico del centro urbano. Condiciones sociales de vida y participación ciudadana ante la política de rehabilitación urbanística del Casco Vello de Vigo».
} 
that 'historical centres' have as an empirical object for urban sciences and as a limited object of intervention for urban policies, I try to concibe the evolution of the concept with a process of «desconstruction». The evolution percibed goes from the urban renewal to several steps of conservationism, firstly, social stigmatization, secondly, and «strategic» and marketing product finally, in coherence with the economic restructuration in global cities.

In support to this conception of 'historical centres' I expose a comparaison between several cities. One of the conclusions adopted implies, firstly, that there is no copies between contemporary urban interventions in historical centres of different cities in relation to consider social dimensions into rehabilitation planning, and, secondly, that is very usual to simplificate the historical, social and spatial heterogeneity of these areas.

Key words: Sociology, Urban Policies, Rehabilitation, Historical Centres.

\section{Introducción: ¿hacia dónde va el centro histórico del centro urbano?}

En los últimos años estamos asistiendo a procesos de rehabilitación urbanística de los centros históricos de muchas ciudades en reestructuración. Pero, ante todo, la rehabilitación es un discurso y éste viene a configurar un imaginario retórico donde caben desde justificaciones de políticas blandas de renovación urbana, hasta mecanismos decisionales muy jerárquicos que constantemente hacen gala pública de ofrecerse bajo un suelo de consenso y participación social desconocidos, se dice, con anterioridad.

No obstante, la problemática que se aloja en los centros históricos en general es más variada y los estudios sociológicos tienden a simplificarla en censos demográficos y reproducción de estereotipos sobre la planificación urbanística más adecuada para combatir las desviaciones sociales. Los cascos antiguos de ciudades de tamaño medio y de metrópolis muestran muy claramente la superposición de segregaciones sociales de clase con demarcaciones culturales de vecindarios, ya que en ellos residen formas de relación social y de ocupación muy resistentes al cambio (varias generaciones de inquilinos que no se van, familias que se ayudan mutuamente, etc.). El cambio en estas áreas, de cualquier modo, viene impuesto por las estrategias de la propiedad inmobiliaria y por la reestructuración del capital en las ciudades industrializadas, que se traduce en una movilización obligada para las clases subalternas, una expulsión del centro urbano, lenta pero eficaz. La despoblación no es un hecho natural y necesario que acompañe a la noción de 'centro histórico, sino se entiende dentro de los procesos de reestructuración urbana que la enmarcan. De hecho, hay diversos mecanismos para consolidar la movilización social forzada, pero los fenómenos de desplazamiento y sustitución a menudo son tan invisibles que se llegan a asumir como inevitables incluso por todos los agentes sociales que participan en la gestión de los planes de rehabilitación.

Las políticas urbanísticas de rehabilitación del centro histórico que he analizado seleccionan arbitraria e interesadamente el tipo de dimensiones sociales a tratar, para subordinarlas a otros intereses. Se eliminan las más molestas, como la problemática interna de las viviendas en alquiler o la segregación por clases y subáreas del casco, y se cubre con discursos y prácticas sociales de participación la continuidad histórica de modelos verticales de planificación urbanística. Por eso los discursos y el sentido de los conceptos de 'centro histórico' me parecen tan relevantes. Las causas de la etiquetada «degradación» de los cascos antiguos no se pueden buscar, pues, sino en el conflicto de definiciones de las condiciones sociales prioritarias sobre las que incide la redistribución urbanística. En este conflicto participan las organizaciones vecinales y los expertos de todo tipo, tanto sociólogos como arquitectos o asistentes sociales, pero quedan numerosos sectores que también 
habitan los espacios históricos, marginados ahora del proceso de toma de decisiones sobre el nuevo diseño de su entorno. La heterogeneidad social, pues, es constreñida por el lenguaje de diseño y gestión que se da realmente en los centros históricos.

El objeto 'centro histórico' tiene su propia existencia real, pero hay que mirarlo desde varios ángulos (porque tiene distintas realidades) y definirlo en base a algún tipo de criterios, o sea, construirlo teóricamente, porque de lo contrario es como si no existiera (para quien intenta conocerlo).

\section{Situando la rehabilitación urbanística en su historia: un esbozo de desconstrucción}

¿Por qué necesitamos una retrospección histórica para fundamentar un análisis del urbanismo rehabilitador del centro histórico? ¿Qué tipo de «historia» se buscará, cuestionará y reconstruirá para explicar los conflictos sociales del centro histórico? Entre estos conflictos está el propio uso de «lo histórico» por distintos agentes urbanos —especialmente técnicos, planificadores, dirigentes vecinales y medios de comunicación localpara justificar u oponerse a las actuales políticas de rehabilitación. Pero también están los olvidos, las censuras sobre el pasado en lo referente a las huellas de movilizaciones sociales anteriores, el significado social que el crecimiento demográfico y metropolitano de la ciudad tenía para su centro histórico.

«La pregunta por el sentido de la historia no tiene, para Foucault, lugar donde plantearse: no tiene sentido. (...) Lo que importa entonces no es la pregunta por el sentido, siempre forzosamente cómplice con el orden actual, siempre encubridora de una necesidad de reconocimiento, sino la pregunta por el funcionamiento de los diferentes avatares históricos que nos empujaría al conocimiento de éstos: al análisis del modo como se oponen, se anudan o se suceden los diferentes acontecimientos - discursivos, institucionales - que acaban por articular el campo de una problemática: una idea (la locura, la enfermedad, el hombre...)» (Morey, 1987, p. 50).

$\mathrm{K}$. Lynch ha ofrecido una caracterización general sobre las contradicciones del «conservacionismo histórico» y, a la vez, un conjunto de razones alternativas para defenderlo. Su planteamiento del problema puede también introducirnos en nuestra particular inmersión en la historia urbanística y social de los centros históricos.

«El mantenimiento del entorno histórico tuvo, en sus comienzos, motivaciones políticas. En Estados Unidos, este movimiento empezó inmediatamente antes de la Guerra Civil, como signo de la ansiedad reciente por impedir al principio y luego por sanar la desastrosa brecha que se abría en la unidad de la nación. Más tarde, el mantenimiento del entorno histórico se conectó, de un modo explícito, a la 'americanización' del inmigrante extranjero que venía a profanarlo. Posteriormente fueron añadiéndose nuevos motivos: corrección en las restauraciones arquitectónicas, investigaciones arqueológicas y, por último, atracción turística según que el disfrute de los lugares históricos se iba convirtiendo en fenómeno generalizado. Hoy en día, este placer se ha tornado en un gusto tan prestablecido que ha dado lugar a que zonas urbanas enteras se hayan preservado y restaurado, de acuerdo con el estado en que se hallaban en alguna época anterior, y no ya sólo para los turistas, sino para los propios residentes» (Lynch, 1981, p. 186). 
Las políticas recientes de rehabilitación han llevado el signo del «conservacionismo», pero esta idea es mucho más antigua. Si comparamos el proceso de su apropiación en los Estados Unidos con lo ocurrido en Europa, podremos enmarcar mejor nuestro objeto de investigación. Posteriormente continuaremos desarrollando el planteamiento de Lynch.

En efecto, tomando casos como los de Francia, Italia y España, podemos observar un esfuerzo legislativo desde el siglo XIX por poner ciertos remedios a un proceso de destrucción de elementos monumentales, artísticos y arquitectónicos, prolongado hasta nuestros días. Lo que es más difícil de interpretar unívocamente es que sólo existiesen «motivaciones políticas» en la valoración urbanística y protección legal de esos elementos.

«Cuando a partir del primer tercio del siglo XIX se hicieron inaplazables las operaciones de ensanche y reforma interior de las ciudades, la burguesía industrial y las nuevas Administraciones locales las afrontaron desde la premisa de la economía de mercado, sacrificando las exigencias de orden estético y la conservación de la ciudad tradicional como legado histórico. El 'ornato' y el 'embellecimiento' de las ciudades pervivieron como objetivos marginales de la policía urbana, cuya prevalencia dependía más de la sensibilidad de las clases dirigentes que de su prescripción legal» (Bassols, 1989, p. 146).

Junto al crecimiento industrial y espacial de numerosas ciudades españolas a principios del siglo XX y en tiempos de la República (Primera y Segunda), surgió también todo un planeamiento estatal que integraba la tutela estatal sobre el «arte colectivo»: la Ley sobre Monumentos Nacionales de 1915, el urbanismo municipalista del Estatuto de 1924 (ver Dávila, 1991, p. 103), el Decreto-Ley de 1926 (con la que se declara que «el Tesoro Artístico por razones de 'arte y cultura' queda confiado a la tutela y protección del Estado, desarrollando un minucioso sistema de protección que afecta también a los Conjuntos histórico-artísticos, ciudades y pueblos artísticos y bellezas naturales», Bassols, 1989, p. 148) y la Ley de 1933 (en el mismo año en que se redactaba la «Carta de Atenas»: biblia del racionalismo moderno que desplazó la influencia que tenían el naturalismo y el proteccionismo de las «Ciudades Jardín» sobre el Estatuto Municipal de 1924).

Estamos, pues, en un período prebélico en España de fuertes oposiciones de bloques políticos e incipiente desarrollo urbano-industrial que va a ser el caldo de cultivo de la noción eficaz de «Centro Histórico» como espacio a conservar. La eficacia, como concepto-instrumento de reacción ante la evidente decadencia de los centros históricos, vendrá dada con toda una serie de movimientos culturales posteriores:

«La aplicación de los principios de la Carta de Atenas en el contexto de la reconstrucción postbélica [en Europa] y el expansionismo económico de la década de los cincuenta van a determinar el gigantismo urbano común a los diversos países industrializados, la fragmentación de la ciudad entre su núcleo preexistente y las nuevas periferias como meros agregados de edificios y espacios sin contenido e identidad. Precisamente, en este contexto de oposición entre la centralidad y la periferia urbanas, va a emerger la problemática de los Centros Históricos de las ciudades. Como producto de un doble movimiento de ideas: la valorización de los Centros Históricos como símbolos de identidad y de condensación de la imagen focal de la comunidad urbana frente a la despersonalización de las periferias; y como defensa contra los intentos de someter las áreas urbanas históricas al dominio de las fuerzas económicas que pretenden destruir el tejido histórico y sustituirlo por actividades terciarias» (Bassols, 1989, p. 150). 
Este nuevo interés, recién despertado a la divulgación internacional de un amplio consenso y, como señalábamos, incubado en tiempos más duros prácticamente pero más preocupados teóricamente, va procediendo mediante saltos. La única legislación para el Estado español, la Ley del Suelo de 1956, y los distintos congresos internacionales (Carta de Venecia, 1964; Coloquios de Quito, declaración de Bolonia, Carta de Amsterdam, 1975; Recomendación de la UNESCO en Nairobi, 1976, etc.) dan lugar, específicamente, a una reacción anti-moderna contra la «renovación» de la ciudad heredada. El concepto que las experiencias europeas y la legislación española de 1985 (Ley del Patrimonio Histórico Español) vendrán a reforzar es el de «rehabilitación integrada». Es decir, una integración del centro histórico consigo mismo, con lo que fue en el pasado. Una integración en el centro urbano como modo de resolver el conflicto de éste con los nuevos cinturones rojos, obreros, surgidos en la periferia urbana. En cierto modo, el conservacionismo rehabilitador venía a suponer un «aislamiento» con respecto a los problemas del desarrollo capitalista de la ciudad (López Sánchez, 1986; Gavira, 1995), pero esta pauta no será absoluta en toda Europa: aparecerán intervenciones críticas en Italia y Portugal, mientras se consolidan rehabilitaciones capitalizadoras en el resto de ciudades europeas y se producen muy lentamente programas de rehabilitación en las ciudades españolas (Dávila, 1993; AA.VV., 1981; Benito, 1989; García Nart, 1989).

Estos procesos no consisten, pues, exclusivamente en construcciones retóricas eficaces. Conectando con lo ocurrido en los años más recientes, podemos retomar la exposición de Lynch para valorar algunas de sus implicaciones teóricas y prácticas:

«Con más frecuencia que con menos, se trata de nuevos residentes atraídos por la calidad histórica de la zona. El mercado ha respondido a la demanda con un rápido aumento del valor de la propiedad. Los antiguos residentes con ingresos bajos o modestos son reemplazados por aquellos que tienen capacidad suficiente para pagar los nuevos e hinchados precios y para quienes la calidad histórica bien lo vale. (...) Las cosas, primero, son nuevas; luego, pasadas de moda, ajadas, despreciables, y, por fin, más tarde, renacen como históricas. La onda del redescubrimiento sigue al presente a una distancia suficientemente prudencial como para que permita el necesario desbroce. Ese intervalo, hoy, parece estar acortándose: antes era de cien años y, ahora, se está acercando a los treinta o cuarenta» (Lynch, 1981, p. 186).

Podemos recapitular algunos de los focos generadores del objeto «centro histórico» y de los valores de «rehabilitación»: a) los intereses políticos de identificación nacional; b) los intereses académicos de redescubrimiento; c) los intereses económicos de explotación turística; d) el consenso cultural ante el prestigio de la conservación de lo antiguo; e) la subordinación de valores estético-arquitectónicos al desarrollo industrial y comercial con la urbanización de los «ensanches»; f) la influencia de teorías urbanísticas en planeamientos variables según su aplicación y contextos políticos; g) el movimiento académicocultural internacional contra la destrucción de las ciudades históricas; h) las contradicciones entre centro urbano y periferias obreras; i) la «gentrification» (desplazamiento de clases populares y sustitución por clases altas) de los cascos rehabilitados; j) los conflictos sociales entre grupos por definir los elementos y períodos con carácter de «histórico».

El hecho de poder contextualizar la evolución de las ahora novedosas políticas de rehabilitación urbanística en los centros históricos nos conduce por varias vías. En primer lugar nos exige argumentar por qué es más histórico un lugar que otro y para qué colectividad, en un contexto social determinado (político, cultural, etc.). En segundo lugar se 
puede insistir más y analizar qué influencias tienen colectivos sociales determinados con privilegio para definir las políticas de rehabilitación (técnicos, arquitectos, etc.) y para implementarlas (administraciones locales, organismos internacionales, etc.). En tercer lugar: ¿qué valoración sociológica se puede hacer de esta historia para orientar las movilizaciones sociales en torno a los centros históricos? Continuemos, pues, viendo las respuestas de Lynch que ofrece, sobre todo, pautas interpretativas y operativas para el gremio de los urbanistas:

«La muy generalizada noción de que las cosas viejas cuando fueron mejores fue en su momento originario, a partir del cual han ido sufriendo un gradual proceso de deterioro (por mucho que esta teoría quizás suponga paralelamente una contradictoria valoración positiva de las cosas que hoy son nuevas), viene a hacer el conflicto todavía más agudo. El convencido de esta teoría tiende a restaurar el objeto preservado, devolviéndole su pureza primitiva y a ocultar cualquier signo de funcionalidad moderna que en él pueda darse, del mismo que el apóstol de la naturaleza intenta camuflar la presencia del hombre en ella. La Historia se detuvo para ellos, en alguna edad de oro, y todo cambio siempre es una torpeza $(\ldots) »$.

Se podría, por tanto, criticar el movimiento en favor de la preservación histórica desde tres supuestos relacionados entre sí. En primer lugar, se puede aducir que la preservación histórica, a menudo, supone el desplazar a las gentes que viven en las áreas que van a ser restauradas. En segundo lugar, que transmite una visión estática, purificada y falsa de la Historia, una visión vívidamente aprehensible, cabe decir, aunque no por ello menos falsa. Por último, se podría añadir que los valores en que se basan los actuales criterios de preservación histórica son excluyentes y especializados [los exteriores deben restaurarse, mientras que los interiores poco importan]. La preservación a gran escala, además, disminuiría el grado de adecuación a nuevas funciones, impidiendo asimismo, futuras adaptaciones». (Lynch, 1981, pp. 186-187).

La rehabilitación actual es heredera de las dos versiones subyacentes de la restauración y del conservacionismo descritos: por un lado, críticamente, en cuanto que corrientes elitistas y románticamente reaccionarias contra las necesarias funciones modernas del entorno urbano (consecuencias: insensibles socialmente con las clases bajas residentes e irracionales para la planificación territorial a gran escala, insostenible con criterios conservacionistas); por otro lado, positivamente, como incremento de la variedad de formas y funciones cuando la conservación es incluyente de interiores y exteriores, y cuando, lejos de la especialización restauradora de las formas originarias, se adaptan algunos elementos y barrios históricos a las diversas funciones modernas.

Algunos investigadores han relacionado fácticamente, por ejemplo, las políticas de rehabilitación italianas de los años setenta y ochenta, con una recuperación del sector de la construcción inmobiliaria (Di Battista, 1989, p. 15). Se trata del llamado crecimiento centrípeto (hacia el centro) cuando está en crisis la expansión urbanizadora centrífuga (hacia el extrarradio) (García Bellido, 1979; Álvarez Mora, 1980). Pero lo interesante de las notas anteriores no es tanto el olvido como la asunción explícita de un urbanismo rehabilitador adaptado al desarrollo capitalista de la ciudad como algo (históricamente) necesario e imparable. La plurifuncionalidad moderna imperante (y «deseable» para la teoría normativa de Lynch) es el referente histórico al que las clases dirigentes que planifiquen la rehabilitación no podrán sustraerse. El hecho de pensar lo histórico, más que 
la Historia, nos ha conducido a dos nuevos focos generadores de las nociones en las que indagamos, sin los cuales, creo, no se puede llegar a una valoración sociológica: k) los grupos residenciales son un objeto más a tener en cuenta por las intervenciones de rehabilitación; 1) los expertos técnicos son algunos de los sujetos de la rehabilitación, y se encuentran divididos según posturas artístico-historicistas o funcional-progresistas, entre otras.

Desconstruir las ideas, las prácticas, las instituciones y los objetos que nos parecen naturales, fijos, definidos, concretos, actuales, no es acabar con la historia, sino devolverle su viveza y dinamismo. De hecho, tanto en los planteamientos más racionales como en los más razonables, nos exige una cierta circularidad y preguntarnos también por el por qué de la historia, por su arbitrariedad lógica. Por eso es preciso responder también reconstruyéndola.

\section{La intertextualidad del centro histórico: la historia cruzada de rehabilitaciones en diferentes cascos antiguos}

La noción de 'centro histórico' no sólo precisa de debates teóricos sino también de ejemplos y experiencias prácticas en las que apoyarse. De este modo, unas rehabilitaciones urbanísticas se apoyan en otras precedentes, adaptan su programación e implantan idénticas o similares soluciones. Podría pensarse que con un amplio bagaje histórico de experiencias de rehabilitación en cascos antiguos, cada nueva rehabilitación partiría ventajosamente de una evaluación del urbanismo pasado, de sus consecuencias acumuladas y de las virtualidades sopesadas que abría. Pero esta evaluación no se produce explícitamente ni con profundidad en la mayoría de casos. En lugar de enriquecerse la noción de "centro histórico', las situaciones de necesidad de cada política de rehabilitación generan nuevos problemas que la cuestionan.

Entre los problemas hallados al revisar los precedentes de rehabilitación se pueden señalar, por lo menos, seis:

a) las dificultades de comparabilidad entre ciudades con desigual desarrollo industrial, institucional, demográfico y de interés (económico y cultural) por sus conjuntos históricos;

b) las contradicciones entre las intervenciones privadas y públicas en la rehabilitación, por carecer de Normativa o por aplicarse con flexibilidad la existente;

c) los diferentes grados organizativos de las intervenciones en los modelos públicos, en cuanto a la financiación, las prioridades de intervención y la participación interadministrativa;

d) las diferentes características de los grupos de población afectados por las rehabilitaciones, los efectos de éstas y la participación social en ellas;

e) los estudios, evaluaciones y publicaciones que registran aspectos parciales de cada experiencia en contradicción con el carácter informativo global que posee;

f) la censura de la presión social, la autoorganización vecinal y la originalidad de las propuestas no institucionales que incidieron en el proceso de rehabilitación urbanística.

$\mathrm{Ni}$ las referencias sobre experiencias internacionales ni los estudios sobre las experiencias dentro del Estado recogen totalmente estas problemáticas, lo cual no significa que no se pueda componer un mínimo marco orientativo rastreando dichas cuestiones en el material disponible. Este material está compuesto de textos: textos técnicos, que se cruzan y se omiten mutuamente, que enfatizan generalmente más lo urbanístico que lo social de las 
intervenciones. Textos que también aportan categorías y variables útiles para la comparación, pero que, en cuanto al velamiento que operan de las contradicciones sociales y políticas en cada rehabilitación, nos obligan a remitir a otros textos no institucionales o fuera de la estricta disciplina urbanística.

La primera comparación general suele hacerse entre Estados Unidos y Europa. Se considera que el movimiento a favor de la rehabilitación comienza antes en Europa, mientras que en Estados Unidos los principios de «renovación urbana» adquieren rango de ley en la década de los cincuenta. No ocurre, sin embargo, que se carezca de precedentes europeos de renovación urbana con demoliciones, reconstrucciones o apertura de viales en la ciudad tradicional y especialmente en los períodos bélicos, postbélicos y de empuje industrializador. Tampoco ocurre que las ciudades norteamericanas carezcan de historia y de problemas de vivienda (contención de los costes residenciales de reproducción de la fuerza de trabajo y garantía de rentabilidad del capital inmobiliario, según Piccinato, 1983, p. 16) en las áreas más antiguas de la ciudad. Lo que es más destacable en cuanto a los contrastes es que la renovación de los centros en uno y otro lugar se acompaña de justificaciones ligeramente diferentes. Los argumentos «museísticos» e «higienistas» son más propios en los casos europeos, enfocando las remodelaciones hacia la mejora de la salubridad, el saneamiento, la iluminación y la aireación de la edificación antigua y la urbanización viaria (ya se trate de recintos medievales o de otros posteriores, es lo de menos). En los casos norteamericanos, desde las primeras recetas a principios de siglo de la Escuela de Chicago, funcionan con más frecuencia los argumentos explícitos de necesidad de la «expansión urbana» y de «orden social», ante la concentración de ilegalismos, delincuencia e inmigración en los guettos centrales. De hecho, durante la década de los sesenta, la expulsión de colectividades pobres, hispánicas y negras de los centros urbanos en renovación, dio lugar a fuertes movimientos de protesta y participación en el planeamiento, convirtiendo al «corazón de la ciudad en el escenario de la lucha política y social» (Bassols, 1989, p. 151).

Robert Goodman lo expuso con crudeza:

«El plan gubernamental compensatorio de realojamiento no fue más que una farsa. Si la legislación de 1949 para la renovación urbana autorizaba la construcción de 810.000 unidades de alojamiento estatal durante los seis años siguientes, en 1967, dieciocho años después, sólo la mitad de esa cifra había sido construida. Pero lo que se había conseguido bajo la bandera de la renovación urbana supuso, para muchos, una dura lección de vida -400.000 viviendas, la mayor parte ocupadas por gente de escasos ingresos, fueron demolidas en las áreas de renovación urbana-. En esas mismas áreas, sólo 107.000 unidades de alojamiento fueron construidas. Y de las construidas sólo 11.000, menos del 3 por ciento del total destruido, lo fueron para uso de la gente pobre» $(1977$, p. 83).

La lucha social que se generó entonces contra el desplazamiento de los cascos antiguos (Hartman et al., 1982) presentaba también coincidencias con la mantenida en los cascos europeos. En ambos, el desarrollo urbano de renovación se ha combinado, desde los años setenta y ochenta, con estrategias de rehabilitación orientadas a la sustitución poblacional de las clases bajas por otras de rentas más elevadas (el fenómeno conocido como «gentrification»). La sociología de la «gentrification» reconoce con refinamiento las etapas de la sustitución socioeconómica: suburbanización, desinversión institucional, abandono de la propiedad y punto de reinversión (Carpenter y Lees, 1995; Álvarez Mora y Roch, 1980). Las intervenciones públicas se han combinado planificadamente con intervenciones priva- 
das (Fainstein, 1994). Dentro de estrategias capitalistas postfordistas, también la renovación y la rehabilitación se han hecho flexibles, por lo que la convergencia europeanorteamericana viene dada desde finales de los años ochenta y durante los noventa, por los «planes estratégicos».

Gavira (1995, pp. 7-10) resume algunos de los rasgos de este nuevo modelo originado en la Escuela de Negocios de Harvard (EE.UU.) en los años 80 y extendido desde 1985 a Milán, Birminghan, Rotterdam, Barcelona, Madrid y, por ejemplo, en los últimos años a Vigo. «En este tipo de planificación se traslada a la ciudad la imagen de la empresa como modelo (flexible, maleable, dinámica y eficaz), transformando al municipio en un paquete de activos que constituye un producto enfrentado a la competencia de otras ciudades» (ibid., p. 7). Se precisa una «imagen de marca» y un «alcalde-líder» que cohesionen una nueva identidad colectiva basada en nuevos circuitos de ocio privado y de consumo por las «calles de moda», en la que se integra toda intervención en los centros antiguos (ibid., p. 9; para una defensa de la planificación estratégica ver Marcelloni, 1994). Se trata, como se señala en el Plan Estratégico de Vigo y su Ârea de Influencia (PEVA), de que

«las ciudades más importantes de Europa se agrupen en 'lobbies' o colectivos que buscan atraer inversiones multinacionales que desarrollen sus áreas de influencia. Los denominados 'Eurometrópolis' y 'Eje Atlántico' son dos manifestaciones de esta tendencia. Para que esta actuación conjunta se produzca es necesario proyectar una imagen global de la ciudad que sea reconocida en el exterior. Pero ello es coyuntural y poco consistente si no va acompañado de una percepción positiva por parte de los propios vecinos, que sienten orgullo de pertenecer a un determinado territorio. (...) Por ello, un plan estratégico debe saber encontrar aquellos puntos fuertes que sepan definir los rasgos constitutivos de la imagen global de la ciudad y su región urbana» (Souto et al., 1994, p. $357)$.

Los años de posguerra fueron propicios en Europa para concebir los centros históricos como Bienes Culturales, superando las concepciones meramente urbanísticas o ambientales.

«El término 'Bien Cultural' aparecerá, por primera vez, en 1954 en la Convención para la Protección de los Bienes Culturales en caso de Conflicto armado. (...) La Comisión Franceschini (1964) incluirá en sus formulaciones como bienes culturales a los Centros Históricos, concebidos como 'aquellas estructuras de asentamiento urbano que constituyen una unidad cultural o una parte original y auténtica de asentamiento que testimonian los caracteres de una cultura urbana viva'» (Bassols, 1989, p. 153).

Las experiencias de rehabilitación acaecidas se caracterizaron generalmente por su conservacionismo tanto de las edificaciones con mayor valor artístico, como de la función residencial (Álvarez Mora y Roch, 1980, pp. 203-214: fijándose en casos franceses, alemanes y polacos), si bien por entonces se iniciaron las tendencias de expulsión y sustitución de las clases populares que habitaban los centros históricos.

En Francia las rehabilitaciones de los cascos desde los años cincuenta se acompañó de amplios programas de vivienda social (financiados públicamente) en barrios periféricos que en años posteriores mostrarán la creciente marginación de la población desplazada de este modo (AA.VV., 1990; Lojkine, 1981). Por contra, las intervenciones italianas y 
portuguesas se destacarán por el movimiento inverso: un intento de planificación rehabilitadora integral y una amplia repercusión de las reivindicaciones ciudadanas en los modelos de gestión.

Campos Venuti (1981) destaca positivamente la participación de «consejos de barrio» en el planeamiento (pp. 59-64) y la perspectiva globalizadora de éste que se intentó aplicar en la zona norte de Italia: descentralización, disminución de densidades funcionales, reducción de usos intensivos, mantenimiento de funciones productivas en el centro urbano, estudio de tipologías y graduación de niveles de conservación, planes de circulación y transporte, y de equipamientos. Entre los elementos prácticos que marcaron escuela de las rehabilitaciones en Bolonia, Pavía y Emilia-Romaña podemos recoger aquellos que enfatizan el carácter social del urbanismo: la legislación sobre alquileres justos y expropiación por absentismo (pp. 153-4), la limitación de la expropiación a los grandes propietarios y la prioridad de convenios con la pequeña propiedad (pp. 146, 170), la creación de viviendas sociales y de «aparcamiento» o provisionales durante las obras de rehabilitación (p. 150) y el soporte de servicios sociales complementarios a la realización de las obras. No deja de ser la visión de un técnico participando en una política urbanística de izquierdas en la que los consejos ciudadanos no siempre participaban de un modo totalmente representativo y decisorio, aunque pusieron alto el listón para intervenciones posteriores (Cervellati et al., 1977).

«En 1969 se efectúa una huelga general nacional en torno a la vivienda. En muchas ciudades del norte comienzan a extenderse los impagos de alquileres, las ocupaciones de viviendas... El 'derecho a la ciudad', la 'vivienda como servicio social', 'la casa se ocupa', 'el alquiler no se paga', son slogans corrientes en aquellos años de extensas y agudas reivindicaciones sociales» (Di Battista, 1989, p. 11).

Y será, pues, a finales de los años setenta cuando las políticas pioneras de rehabilitación tendrán más auge y se articularon bajo la intención política de superar la contradicción entre «infrautilización» $\mathrm{y}$ «deterioro»:

«Se comprueba que las condiciones de deterioro de la edificación no mantienen una relación lineal con la antigüedad sino que el deterioro se produce sobre todo en períodos iniciales prosiguiendo después más lentamente (el umbral del deterioro máximo coincide aproximadamente con los cuarenta años de vida del edificio). Por otra parte, las relaciones entre condiciones socioeconómicas de los residentes y edad y estado de conservación de los edificios refleja una elevada concentración de los sectores menores de renta en los edificios de más de cuarenta años y más deteriorados» (Di Battista, 1989, p. 12).

En los años ochenta, sin embargo, todo ese urbanismo «comprometido» dará un giro de retroceso ante las críticas que recibió por los elevados costes de sus intervenciones y por la falta de especialización de métodos de recuperación de lo antiguo (Di Battista, 1989, pp. 14-15). Las contradicciones, pues, se agudizan con la privatización en las recuperaciones, en los servicios urbanos y en la especialización terciaria (Piccinato, 1983, pp. 23-25), mientras que las críticas especializadas se hacen menos radicales políticamente, volviendo a aspectos básicos de la teoría urbanística: complejidad del equilibrio funcional en los centros históricos; valores pedagógicos de la ciudad histórica, de su trazado y su plano; equiparación de importancia histórica entre la ciudad preindustrial y la del siglo XIX, etc. (Cerasi, 1990, pp. 46, 55). 
En Portugal los proyectos SAAL (programas estatales de intervención en barrios degradados: Servicios de Apoyo Descentralizado Local) tuvieron su inicio con la revolución de 1974 y tocaron de lleno las necesidades de vivienda de las áreas marginales, entre las que estaban los centros históricos. Combinando instrumentos estatales de expropiación, financiación, asistencia social y técnica y viviendas sociales con comisiones participativas de barrio, obtuvieron una fuerte repercusión en las grandes ciudades y algunos proyectos de rehabilitación se han continuado durante varias décadas, como es el caso de Oporto (De Oliveira y Marconi, 1978; Siza, 1977). La participación pasó a ser «algo obligado» (Portas, 1983 , p. 63) y se introdujo especialmente una nueva relación entre bases ciudadanas y técnicos: el SAAL

«a grandes rasgos consistía en ofrecer a los movimientos vecinales la oportunidad de transformarse en promotores de vivienda y de mejorar los barrios existentes sin cambio de lugar en la ciudad, es decir, reconocerles el derecho a quedar en el sitio donde estaban y a gestionar la operación. Dicha operación podía incluir desde la gestión directa hasta la autoconstrucción pasando por la contratación de empresas de construcción (...) Creaba la posibilidad de que las poblaciones organizadas, si estaban interesadas, podían elegir formas de autogestión del barrio ya que los contactos con los arquitectos, empresas y Ayuntamiento los hacían los vecinos y no el IPPV portugués. Este sólo se encargaba de pagar la factura, hasta que los gobiernos posteriores han recortado los presupuestos» (Portas, 1983, p. 63).

No obstante, los arquitectos empezaron a abandonar las oficinas municipales y comarcales en los primeros ochenta, rentabilizando en la profesión privada su experiencia urbanística (ibid. p. 65). Además, los SAAL no lograron alterar la valorización capitalista del suelo ni la jerarquización en la que los técnicos se situaban ambiguamente en los procesos participativos y asistenciales, por lo que a pesar de las iniciativas públicas se reprodujeron las desigualdades de clase y residenciales (Matías, 1975, pp. 38, 48).

En el Estado español disponemos de algunas obras recientes haciendo balance de las experiencias de los años ochenta en adelante ya que, en cierto sentido, suponen una continuidad con las concepciones importadas del debate internacional anterior. Por otra parte, la intertextualidad se complica cuando para cada ciudad hay estudios y registros muy desiguales de cada intervención según el tipo de artículos, memorias o investigaciones publicadas.

Al mismo tiempo, los cascos históricos encuentran un doble marco jurídico nuevo: primero con la ampliación de la política de vivienda a la rehabilitación del patrimonio arquitectónico, desarrollándose las Áreas de Rehabilitación Integrada (1983); después, con la Ley del Patrimonio Histórico español (1985) que regula la declaración y protección de Bienes de Interés Cultural y de Conjuntos Histórico-Artísticos (Bassols, 1989, pp. 153166). Esto indica la influencia, tardía pero efectiva, del movimiento cultural en pro de la recuperación arquitectónica y de la mayor cobertura legal de los Planes Especiales que generalmente se redactan para los centros históricos. «Se han obtenido éxitos significativos en la defensa del patrimonio edificado, especialmente en las arquitecturas monumentales y singulares, no tanto en las de tipo ambiental y en los aspectos sociales y funcionales» (Troitiño, 1992, p. 39). En 1989 existían 333 conjuntos históricos declarados, 254 con el expediente de declaración incoado y hasta un total de unos 1.200 que podrían ingresar en tal categoría (ibid., p. 48), si bien según el rango y el poder de cada ciudad, sólo en contados casos se desarrolla la legislación adecuada. 
En los primeros estudios compilados por el MOPU (AA.VV. 1981, 1982) se observa la tendencia a codificar las condiciones sociales de habitabilidad y ocupación de los centros históricos mediante unos mínimos datos demográficos y cifras de distribución residencial. En teoría se describe una situación social y urbanística de fuerte «deterioro» y «degradación» para los centros urbanos y los núcleos rurales, aunque luego las variables sociales usadas restringen mucho la caracterización de esa situación (cuantificando simplificadamente lo social). La situación, así, se concibe como: ocupación de

«clases económicamente débiles y marginadas y abandono de las capas de mayores niveles de renta; habitabilidad insatisfactoria, deficiencias estructurales, falta de servicios y hacinamiento; desaparición de la vida social, despoblamiento, delincuencia, ausencia de comercio y de equipamientos» (Lowy, 1982, p. 23); «deterioro físico e infrautilización de la edificación, actividades comerciales y artesanas en decadencia, población residente empobrecida y envejecida, notable persistencia del arrendamiento, escasa actividad constructiva y mercado inmobiliario residual» (Gago, 1982, p. 31).

Todavía se evaluaban provisionalmente más las posibilidades de rehabilitación que experiencias concretas de planeamiento y gestión, si bien se reconocían ya algunos aspectos cruciales de los problemas sociales demográficos, de clase, de financiación, de vivienda y de equipamientos que afectaban a la mayoría de centros históricos incluso antes que fenómenos como la delincuencia (sólo mencionada en dos de los 29 casos estudiados).

Evaluando ya el desarrollo del planeamiento específico de rehabilitación en ciudades de tamaño medio (excepto Barcelona, Madrid, Valencia y Zaragoza: las demás eran Logroño, Vitoria, Salamanca, Tafalla, Estella, Pamplona, Sos del Rey Católico, Almería y Palma de Mallorca), un equipo de investigación posterior llegaba a conclusiones urbanísticas muy detalladas (eficacia de órganos gestores descentralizados, necesidad de estudios económicos-financieros, etc.: García Nart, 1989, p. 34), mientras que socialmente el grado de generalización era mayor: en cuanto a la población establecía una densidad media de 226 Hab./Ha., un peso poblacional con respecto a la ciudad del 8,64\%, y una población activa media del 32\%; en cuanto a la vivienda se halló un media de 108 Viv./Ha., una media del $18 \%$ de viviendas desocupadas y un porcentaje medio de tenencia en propiedad cercano al $50 \%$ (Rubio et al., 1990, p. 30). Dentro de las comparaciones entre las villas gallegas aparece, por poner un ejemplo de contraste, un mayor porcentaje de habitantes propietarios, excepto en las grandes ciudades (González-Cebrián, 1989, p. 93). Tomando como referencia los conjuntos históricos donde se elaboraron Planes Especiales, un estudio de 1983 (?) también establecía unas conclusiones generales para todo el Estado, con los siguientes porcentajes medios de uso y condiciones sociales: en cuanto a la población se halló un peso poblacional con respecto al total de la ciudad del 5-10\%, una dinámica de descenso demográfico entre 1970 y 1990 del -10 a -20\% y unas rentas familiares del 2025\% menores del S.M.I. (Salario Mínimo Interprofesional), del 60-65\% menores de 2,5 SMI y del 30\% entre 2,5 y 4 SMI; en cuanto a la ocupación y vivienda se estableció una ocupación del suelo urbano total del 7-13\%, un uso terciario del $60-70 \%$, una media de viviendas desocupadas de $10-15 \%$, con un $30-35 \%$ de viviendas necesitadas de rehabilitación y un $5 \%$ de edificación ruinosa ${ }^{1}$. Evidentemente, con toda esta información descono-

1 El estudio publicado por el MOPT lleva el título de «Planes Especiales de Protección del Patrimonio Histórico-Artístico. Problemática del reparto de cargas y beneficios», y los datos están citados por Pino (1994, p. 57), de ahí que dudemos de la fecha exacta de publicación (podría ser de 1993, ya que el concepto que se ofrece de dinámica poblacional abarca hasta 1990). 
cemos muchos ejes de la estructura social (estudios, composición familiar, etc.), de los conflictos sociales y de las reivindicaciones, iniciativas, apropiaciones y modos de participar de los grupos comunitarios organizados. La cuestión se amplía si preguntamos: ¿qué ha pasado realmente con esas condiciones sociales en las ciudades donde se han aplicado políticas de rehabilitación?

El universo de respuestas es también aquí muy colorido. Troitiño compara los aspectos históricos y sociales en los casos de Madrid, León y Cuenca, con los de otras ciudades (como Vitoria y Alcalá de Henares) sin llegar a identificar los efectos sociales más directos y concluir con la necesidad de planeamientos integrales (coordinando políticas sectoriales) y de iniciativas financieras estatales más considerables, sobre todo donde hay «grupos sociales que sin ayudas especiales nunca podrán incorporarse a la rehabilitación» (1992, pp. 46-47). Sobre ciudades como Alcoy, Lérida, Gijón, Puerto Real, Oviedo o Santiago de Compostela (Dávila, 1993; Amadó, 1989; Pol, 1989, 1989b; Seguí, 1989; AA.VV., 1993-94; Fernández Fernández, 1995; Campesino, 1989) se pueden encontrar textos que destacan las inversiones económicas en infraestructuras y vivienda, por una parte, y los objetivos más o menos globales de los planeamientos por otra, mientras que los problemas sociales enunciados (infravivienda, tráfico, etc.) se disuelven en los proyectos urbanísticos (en Andalucía, sin embargo, tenemos noticias muy puntuales de lo contrario, con respecto a la recuperación monumental para viviendas sociales: González Tamarit, 1995; en Santiago sobresale también un exitoso y millonario programa de ayudas a la rehabilitación de particulares, tanto de fachadas como de interiores: Fernández Fernández, 1995).

Si tomamos los problemas de la vivienda y de la participación social en el planeamiento, podemos ver cómo en la mayoría de casos los efectos progresistas en estas materias están ausentes. En Barcelona, por ejemplo, se promovió la constitución de una cooperativa de viviendas en alquiler, se rehabilitaron más de 800 viviendas y se realizaron expropiaciones por manzanas que no siempre beneficiaban a los pequeños propietarios (a veces obligando al «esponjamiento»), quedando la participación popular, por las noticias que tenemos, reducida a campañas publicitarias muy populares y a la expulsión de grupos marginales (prostitución, mendicidad, drogadicción, etc.) en los años de las Olimpiadas (Cabrera, 1991; Casas, 1994; Oliveras, 1991; Ribeiro, 1991; López Sánchez, 1986; García Rey, 1994). En Madrid se han producido las inversiones económicas más fuertes y, sin embargo, más parcas en relación a los problemas de infravivienda, alquiler e insolvencia de la población anciana e inmigrante de su amplio centro histórico que es progresivamente expulsada, mientras que, por su parte, la consolidada organización vecinal de la zona ha visto rechazadas muchas de sus elaboradas propuestas de equipamientos y urbanización alternativas, a cambio de los mecanismos de convenios privados (a veces, incluso, pagando a las personas ancianas para abandonar sus viviendas) (Palmero, 1989; Álvarez Mora, 1979; Pol, 1989b; Troitiño, 1992; Busto, 1991; AA.VV., 1994; EMV, 1994; Roch, 1989).

El caso ejemplar de Vitoria por la integralidad de sus planteamientos urbanísticos, económicos y sociales durante más de una década, entre los que solucionaba parte de la vivienda para la población anciana al tiempo que promovía iniciativas laborales mediante escuelas-taller y, decían sus gestores, integraba participativamente a los grupos organizados en el planeamiento, no parece haber solucionado el hecho de ser el casco histórico con mayor índice de pobreza de Euskal Herría al haber dispersado, por ejemplo, según algunos investigadores, a las familias gitanas por otros barrios de la ciudad (Sánchez, 1990; Troitiño, 1992; Manzanos, 1992). En Salamanca también se desarrolló una amplia remodelación del centro histórico con creación de algunas viviendas sociales en su seno, pero con la necesidad de enviar a otras viviendas sociales de la periferia a la gran mayoría, generando una zona muy conflictiva, marginal y segregada; por su parte, los mismos técnicos recono- 
cieron el fracaso de los medios participativos formales que dispusieron y lo limitado decisoria y representativamente de éstos (Contreras et al., 1987; Sosa y Guerra, 1995; Puig Rey, 1994; López Asenjo, 1995). Por último, la también exitosa peatonalización del centro histórico junto a otros proyectos de rehabilitación en Oviedo, según algunas investigaciones, ha ido acompañada de consecuencias residenciales ya conocidas como la expulsión de población autóctona, su sustitución por clases sociales más solventes, la provocación de ruinas, la revalorización y especulación del parque inmobiliario, mientras que la participación social en este caso fue muy diversa y movida fundamentalmente por intereses gremiales (los propietarios contra el catálogo de edificios, los comerciantes contra la peatonalización, los arquitectos contra el conservacionismo y algunos vecinos por salvar edificaciones emblemáticas) (Tomé, 1988; Pol, 1994).

No podemos ahora insistir en la relación de estas contradicciones sociales en las políticas de rehabilitación con el panorama general de la rehabilitación de viviendas en los sucesivos Planes Cuatrienales de vivienda (que, en general, ha sido mínima: Paniagua, 1995; Rehabitec'96, 1996), pero lo que sí queda al descubierto es que, a pesar del reciente desarrollo del urbanismo conservacionista, no existen medidas simultáneas de transformación social en algunas de las vías virtualmente esbozadas aquí.

\section{Bibliografía}

AA.VV. (1981): Estudios básicos de rehabilitación en centros urbanos y núcleos rurales, MOPU, Madrid.

AA.VV. (1982): La rehabilitación integrada del Patrimonio Arquitectónico en centros urbanos, núcleos rurales y conjuntos histórico-artísticos, MOPU, Madrid.

AA.VV. (1990): Quartiers fragiles et développment urbain, Pour, n ${ }^{\circ}$ 125-6, L'Harmattan.

AA.VV. (1993-94): Cuadernos del Camino de Santiago y Voces en Compostela, Consorcio de Santiago de Compostela.

A.VV. La Corrala (1994): Sugerencias Nuevo Plan General de Ordenación Urbana, Informativo AVV Latina-Rastro-Lavapiés, Madrid.

ÁlVAREZ MORA, A. y ROCH, F. (1980): Los centros urbanos, Nuestra Cultura, Madrid.

ÁLVAREZ MORA, A. (1979): Madrid: las transformaciones del centro-ciudad en el modo de producción capitalista, COAM, Madrid.

- (1983): El patrimonio arquitectónico y urbano, Diputación de Madrid, Madrid.

AMADÓ, R. y DOMĖNECH, L. (1989): «Plan del Centro Histórico de Lérida: Barrio del Canyeret», Geometría, no 8, Málaga.

BASSOLS COMA, M. (1989): «Instrumentos legales de intervención en los Conjuntos Históricos», en AA.VV.: Primeras Jornadas de Planeamiento Especial para los Conjuntos Históricos, COAG, Santiago de Compostela.

BENITO MARTÍN, F. (1989): «La protección del Patrimonio en los Centros Históricos», en AA.VV.: Primeras Jornadas de Planeamiento Especial para los Conjuntos Históricos, COAG, Santiago de Compostela.

BUSTO, D. (1991): Política de rehabilitación de viviendas, El Nudo de la Red, $\mathrm{n}^{\circ}$ 17, Madrid.

CABRERA I MASSANES, P. (1991): «Antecedentes urbanísticos», en AA.VV.: La recuperación de una zona urbana degradada: el modelo de Ciutat Vella en Barcelona, Procivesa, Barcelona.

CAMPESINO FERNÁNDEZ, A.J. (1989): «La rehabilitación integrada de los centros históricos: el reto urbanístico de finales de los ochenta», Investigaciones Geográficas, $\mathrm{n}^{\circ} 7$, Universidad de Alicante.

CAMPOS VENUTI, G. (1981, or.1978): Urbanismo y austeridad, S. XXI, Madrid.

CARPENTER, J. y LEES, L. (1995): «Gentrification in New York, London and Paris: An International Comparison», International Journal of Urban and Regional Research.

CASAS, X. y RUEDA, S. (1994): «Ciutat Vella: la renovación integral del Centro Histórico de Barcelona», Alfoz, no 109, Madrid. 
CERASI, M. (1990): El espacio colectivo de la ciudad. Construcción y disolución del sistema público en la arquitectura de la ciudad moderna, Oikos-Tau, Barcelona.

CERVELLATI, P.L.; SACANNAVINI, R. y, DE ANGELIS, C. (1977): La nuova cultura della città. La salvaguardia dei centri storici, la riapropiazione sociale degli organismi urbani e l'analisi dello sviluppo territoriale nell'esperienza di Bologna, Mondadori, Milano.

CONTRERAS, F., ET AL. (1987): Salamanca. Plan Especial de Protección y Reforma Interior del recinto universitario y zona histórico-artística, MOPU-Ayto. Salamanca, Madrid.

DÁVILA LINARES, J.M. (1991): «La ordenación urbanística durante la primera mitad del siglo XX. Premisas para un tratamiento integral de los espacios urbanos», en Investigaciones Geográficas, $\mathrm{n}^{\circ}$ 9, Universidad de Alicante.

- (1993): «La recuperación integrada de centros históricos. Elementos de cualificación espacial en las políticas de desarrollo regional. El caso de Alcoy», en Ciudad y Territorio, $\mathrm{n}^{\circ}$ 98, MOPTMA.

DE OLIVEIRA, P. y MARCONI, F. (1978): Política y proyecto. Una experiencia de base en Portugal, Gustavo Gili, Barcelona.

DI BATTISTA, V. (1989): «Métodos y procesos en la recuperación de la edificación residencial en Italia», en F. Pol (coord.). Ciudad, historia, proyecto, UIMP-MOPU, Madrid.

EMPRESA MUNICIPAL DE LA VIVIENDA (1994): «Normas complementarias para el desarrollo y ejecución del convenio suscrito entre la Comunidad Autónoma de Madrid y el Ayuntamiento de Madrid para la Rehabilitación del Patrimonio Residencial y Urbano», Ayuntamiento de Madrid.

FAINSTEIN, S. (1994): The City Builders. Property, Politics \& Planning in London and New York, Blackwell, Oxford.

FERNÁNDEZ FERNÁNDEZ, P.A. (1995): «Planificación y gestión de la ciudad: la experiencia de Santiago», en Seminario Vivir las ciudades históricas, Universidad de Salamanca.

GAGO LLORENTE, V. (1982): «Por una rehabilitación económicamente viable», en AA.VV.: La rehabilitación integrada del Patrimonio Arquitectónico en centros urbanos, núcleos rurales y conjuntos histórico-artísticos, MOPU, Madrid.

GARCÍA BELLIDO, J. y GONZÁLEZ TAMARIT, L. (1980, or.1979): Para comprender la ciudad. Claves sobre los procesos de producción del espacio, Nuestra Cultura, Madrid.

GARCÍA NART, M. (1989): «Aspectos del Planeamiento urbanístico en los Conjuntos Históricos de España», en AA.VV., Primeras Jornadas de Planeamiento Especial para los Conjuntos Históricos, COAG, Santiago de Compostela.

GARCÍA-REY, J. (1994): «Sevilla: retrato tras la resaca del 92», en Viento Sur, n 13.

GAVIRA, C. (1995): «De la imagen social de la ciudad al símbolo del markéting urbano», en Seminario Vivir las ciudades históricas, Universidad de Salamanca.

GONZÁLEZ-CEBRIÁN TELLO, J. (1989): «Características del Planeamiento de Protección Histórica de Galicia», en AA.VV.: «Primeras Jornadas de Planeamiento Especial para Conjuntos Históricos», COAG, Santiago de Compostela.

GONZÁLEZ TAMARIT, L. (1995): «La política de vivienda, instrumento de recuperación de la ciudad histórica. La experiencia de Andalucía. Algunos casos en Iberoamérica», en Seminario Vivir las ciudades históricas, Universidad de Salamanca.

GOODMAN, R. (1977, or.1971): Después de los urbanistas, ¿qué?, H. Blume, Madrid.

HARTMAN, C.; KEATING, D.; LEGATES, R. y TURNER, S. (1982): Displacement. How to fight it, Legal Services Anti-Displacement Project, Berkeley.

LOJKINE, J. (1981): «Urban policy and local power: some aspects of urban research in Lille», en M. Harlow y E. Lebas (ed.): City, class and capital. New developments in the political economy of cities and regions, Edward Arnold, London.

LÓPEZ ASENJO, A. (1995): «Experiencia de rehabilitación en Salamanca», en Seminario Vivir las ciudades históricas, Universidad de Salamanca.

LÓPEZ SÁNCHEZ, P. (1986): El centro histórico, un lugar para el conflicto. Estrategias del capital para la expulsión del proletariado del centro de Barcelona, Geocrítica, Barcelona.

LOWY, A. (1982): «Aspectos sociales de la rehabilitación», en AA.VV., La rehabilitación integrada del Patrimonio Arquitectónico en centros urbanos, núcleos rurales y conjuntos histórico-artísti$\cos$, MOPU, Madrid.

LYNCH, K. (1985, or.1981): La buena forma de la ciudad, Gustavo Gili, Barcelona. 
MANZANOS, C. (1992): «Desigualdad, pobreza y marginación social en Euskadi», en AA.VV.: La sociedad de la desigualdad. Pobreza y marginación a debate, Gakoa-Tercera Prensa, Donostia.

MARCELLONI, M. (1994): «La planificación estratégica», en F. Herrera et al.: Las grandes ciudades en la década de los noventa, Alfa Delta, Madrid.

MARTÍNEZ, M. (1995): Participación dende abaixo. Espacio social, cultura, economía e política no urbanismo do Casco Vello de Vigo, Cooperativismo e Economía Social, no 12.

MARTÍNEZ, M. (1996): El dominio histórico del centro urbano. Condiciones sociales de vida y participación ciudadana ante la política de rehabilitación urbanística del Casco Vello de Vigo, Tesina de Licenciatura, UCM.

MATÍAS FERREIRA, V. (1975): Movimentos Sociais Urbanos e Intervençao Política. A intervençao do SAAL (Servicio Ambulatorio de Apoio local) em Lisboa, Cidade em questao 4 / Afrontamento, Porto.

MOREY, M. (1987): «Erase una vez...: M. Foucault y el problema del sentido de la historia», en R. Máiz (comp.): Discurso, poder, sujeto. Lecturas sobre Michel Foucault, Universidade de Santiago de Compostela, Santiago.

OLIVERAS, J. (1991): «Obras: rehabilitación de viviendas», en AA.VV.: Ciutat Vella, Procivesa, Barcelona.

PALMERO MARTÍN, M. (1989): «La experiencia madrileña: Los Programas de Intervención Preferente», Geometría, n 7, Málaga.

PANIAGUA, J.L. (1995): «Balance y perspectivas de la política de vivienda en España», en L. Cortés (comp.): Pensar la vivienda, Talasa, Madrid.

PICCINATO, G. (1983): «El problema del centro histórico», en F. Ciardini y P. Falini (eds.): Los centros históricos. Política urbanística y programas de actuación, Gustavo Gili, Barcelona.

PINO VICENTE, D. (1994): «Aspectos socioeconómicos da revitalización dos cascos históricos: ¿sobrebeneficios ou custos engadidos?», Analise Empresarial, nº 21.

POL, F. (1989): «La recuperación del casco antiguo de Gijón», Geometría, nº 8, Málaga.

- (1989b): «La recuperación de los Centros Históricos de España: de las Políticas Urbanas a la Intervención Arquitectónica», en AA.VV.: Primeras Jornadas de Planeamiento Especial para Conjuntos Históricos, COAG, Santiago de Compostela.

- (1994): «Proyectar la rehabilitación urbana: Métodos y estilos», en AA.VV.: El renacimiento de la ciudad contemporánea. Estrategias y procesos de recualificación urbana, IGVS-Europan, Santiago de Compostela.

PORTAS, N. (1983): «Algunos contrastes entre las experiencias urbanísticas portuguesa y española», Obradoiro, $\mathrm{n}^{\circ} 8$.

PUIG REY, P. (1994): «Salamanca: una experiencia en la recuperación peatonal del Casco Histórico», Alfoz, no 109 , Madrid.

REHABITEC'96 (1996): «Las ayudas públicas a la rehabilitación y su nueva plasmación en los Planes de la Vivienda 1992/95 y 1996/99», El Monitor, nº 1786.

RIBEIRO, A.; CLAVELL, D. y CASTRO, I. (1991): Gestión urbanística: liberación de suelo, Ciutat Vella, Procivesa, Barcelona.

RUBIO, J. ET AL. (1990): La política de rehabilitación urbana en España. Evolución, experiencias y efectos, MOPU-ITUR, Madrid.

SÁNCHEZ ÍÑIGO, L.M. (1990): «La rehabilitación del casco medieval de Vitoria-Gasteiz, factor de cohesión social», en Ciudad y Territorio, $\mathrm{n}^{\circ} 85$, Madrid.

SEGUÍ, J. (1989): «Un Plan muy especial para Puerto Real», Geometría, nº 8, Málaga.

SIZA, A. (1977): «Tres intervenciones en la ciudad de Oporto», en A. Rossi (dir.): Proyecto y Ciudad Histórica, COAG, Santiago de Compostela.

SOSA, N.M. y GUERRA, C. (1995): Vivir en la periferia. Estudio sociológico en el barrio de Buenos Aires mediante Investigación-Acción Participativa, Asoc. Cultural Buenos Aires, Salamanca.

SOUTO, X.M. ET AL. (1994): «Sociedad y territorio. Proyectos y frustraciones», en X.M. Mella (dir.): Plan Estratégico de Vigo y su Área de Influencia, Consorcio Zona Franca de Vigo, Vigo.

TOMÉ FERNÁNDEZ, S. (1988): «Oviedo: Un centro histórico en transformación», Ciudad y Territorio, $\mathrm{n}^{\mathrm{o}} 78$, Madrid.

TROITIÑO VINUESA, M.A. (1992): Cascos antiguos y centros históricos: problemas, políticas y dinámicas urbanas, MOPT, Madrid. 